Chirurg 2019 90 (Suppl 2):S98 https://doi.org/10.1007/s00104-019-0901-9 Online publiziert: 13. Februar 2019

(c) Springer Medizin Verlag GmbH, ein Teil von Springer Nature 2019

\section{Originalpublikation}

Swearingen AJ, Kahramangil B, Monteiro R et al (2018) Analysis of postoperative biochemical values and clinical outcomes after adrenalectomy for primary aldosteronism. Surgery 163:807-810

Hintergrund. Bei 5-15\% der Patienten mit Hypertonus ist die Ursache der Hypertension ein primärer Hyperaldosteronismus (PHA; CONN-Syndrom), ausgehend von einem unilateralen Nebennierenrindenadenom oder einer uni- oder bilateralen Hyperplasie. Die Diagnose eines PHA wird gestellt, wenn die Kombination einer erhöhten Plasmaaldosteronkonzentration (PAK) mit einer supprimierten Plasmareninaktivität (PRA) vorliegt und damit eine erhöhte AldosteronRenin-Ratio (ARR) besteht. Eine ARR von 23,6 gilt allgemein als diagnostisch für das Vorliegen eines PHA. Eine chirurgische Therapie ist nur bei unilateraler Aldosteronhypersekretion indiziert, die Leitlinien empfehlen daher zur Seitenlokalisation ein adrenales Venensampling $[1,2]$. Die uneinheitlichen Standards des Venensamplings und das potenzielle Vorkommen einer zusätzlich essenziellen Hypertension erschweren es jedoch, bereits frühpostoperativ erkennen zu können, ob die Adrenalektomie erfolgreich war oder nicht. Hintergrund der vorliegenden Studie war daher zu prüfen, ob

Dieser Beitrag wurde erstpubliziert in Der Chirurg (2018) 89:828-828. https://doi.org/10. 1007/s00104-018-0694-2

\title{
H. Dralle
}

Sektion Endokrine Chirurgie, Klinik für Allgemein-, Viszeral- und Transplantationschirurgie, Universitätsklinikum Essen, Essen, Deutschland

\section{Frühpostoperative Erfolgskriterien der Adrenalektomie beim Conn-Syndrom}

die am ersten postoperativen Tag (POT1) gemessenen Hormonparameter aussagekräftig hinsichtlich des biochemischen und klinischen Outcomes sind.

Material und Methoden. Bei 20 von 44 Patienten mit PHA wurden präoperativ und am POT1 die PAK und PRA bestimmt. Die präoperative PAK betrug im Mittel („mean“) 32,5 ng/dl, die ARR $>137$. Bei allen Patienten erfolgte eine Langzeitkontrolle des Blutdrucks, der Kalium- und spezifischen Hormonkonzentrationen.

Ergebnisse. Insgesamt waren $40 \mathrm{der}$ 44 Patienten im Langzeitverlauf klinisch und biochemisch geheilt, 4 nicht. Bei allen klinisch geheilten Patienten kam es postoperativ zum Abfall der ARR (22-99,6\%). Der ARR-Cut-off von $<23,6$ am POT1 hatte einen positiven prädikativen Wert (PPV) von $95 \%$. Bei den klinisch geheilten Patienten betrug der PAK-Abfall am POT1 33,1 ng/dl, die nicht geheilten Patienten hatten dagegen einen Anstieg der PAK um 3,9ng/dl. Der Unterschied der Hormonwerte zwischen POT1 und Langzeitkontrolle war nicht signifikant. Die Receiver-operating-curve-Analyse ergab für einen PAKAbfall um $10 \mathrm{ng} / \mathrm{dl}$ am POT1 als Cut-off eine Sensitivität von $94 \%$ und Spezifität von $100 \%$ (PPV $100 \%$; NPV 50\%).

Diskussion. Die untersuchte Patientenzahl war relativ niedrig. Es konnte jedoch in der vorliegenden Studie erstmals überzeugend gezeigt werden, dass die ARR- und PAK-Werte bei den klinisch und biochemisch geheilten Patienten bereits am POT1 nach Adrenalektomie in den Normbereich abfallen und bei einem Abfall der PAK um $10 \mathrm{ng} / \mathrm{dl}$ eine Heilung des PHA anzeigen. Für die Patienteninformation und ihre weitere Nachsorge ist damit ein wichtiges Ergebnis erzielt worden.

\section{Korrespondenzadresse}

Prof. Dr. med. Dr. h. c. mult. H. Dralle, FRCS, FACS, FEBS

Sektion Endokrine Chirurgie, Klinik für Allgemein-, Viszeral- und Transplantationschirurgie, Universitätsklinikum Essen Hufelandstr. 55, 45147 Essen, Deutschland henning.dralle@uk-essen.de

Interessenkonflikt. H. Dralle gibt an, dass kein Interessenkonflikt besteht.

\section{Literatur}

1. Rossi GP, Auchus RJ, Brown M et al (2014) An expert consensus statement on use of adrenal vein sampling for the subtyping of primary aldosteronism. Hypertension 63(1):151-160. https://doi.org/10.1161/HYPERTENSIONAHA.113. 02097

2. Funder JW, Carey RM, Mantero F et al (2016) The management of primary Aldosteronism: case detection, diagnosis, and treatment: an endocrine society clinical practice guideline. J Clin Endocrinol Metab 101(5):1889-1916. https://doi. org/10.1210/jc.2015-4061 